\title{
Summability Method for a Horn-Shaped Region
}

\author{
Alexander Moroz \\ Institute of Physics ČSAV, Na Slovance 2, CS-180 40 Prague 8, Czechoslovakia \\ Received February 8, 1990
}

\begin{abstract}
A formulation of the Nevanlinna-like theorem for a horn-shaped region is given. Product of functions obeying the hypotheses of the theorem is also shown to obey these hypotheses, i.e. the summability mechanism preserves nonlinear perturbative conditions such as unitarity of the Feynman series.
\end{abstract}

\section{Introduction}

There is a notoriously known fact that perturbation series in quantum theory are mostly divergent and can have at best the meaning of asymptotic series.

Unlike convergent power series the divergent asymptotic series do not determine a function uniquely. More precisely given an arbitrary sequence $\left\{a_{n}\right\}_{0}^{\infty}$ of complex numbers and an arbitrary sector-like domain $D$, there exists for some $\varepsilon>0$ a function $f(z)$, which is regular in $D_{\varepsilon}:=D \cap\{z|| z \mid<\varepsilon\}$ and such that for every $n$,

$$
\lim _{\substack{z \rightarrow 0 \\ z \in D_{\varepsilon}}}\left(f(z)-a_{0}-\ldots-a_{n} z^{n}\right) / z^{n+1}=a_{n+1}
$$

exists, or equivalently

$$
f(z) \sim \sum_{n=0}^{\infty} a_{n} z^{n} \quad\left(z \rightarrow 0, z \in D_{\varepsilon}\right),
$$

i.e. $\sum_{n=0}^{\infty} a_{n} z^{n}$ is an asymptotic series of $f(z)$ in the region $D_{\varepsilon}[\mathrm{R}, \mathrm{H}]$. In general there are infinitely many functions with the above properties. However, imposing some additional conditions, the so-called strong asymptotic conditions (SAC) on the rest term $R_{N}(z)$,

$$
f(z)=\sum_{n=0}^{N-1} a_{n} z^{n}+R_{N}(z),
$$


the asymptotic series can determine the function $f(z)$ uniquely. As it was shown in [M 2] possible violation of SAC indicates presence of nonperturbative effects in a theory and instability of its ground state. It was emphasized that the violation of $\mathrm{SAC}$ is a more serious problem of a theory than the divergence itself, therefore, the violation of SAC leads to ambiguity of perturbation theory (PT) predictions. When SAC are fulfilled then the coefficients $a_{n}$ of PT determine a $f(z)$ uniquely. To obtain this function an appropriate analytic regular summability method can be used $[\mathrm{H}]$. We say that a summability method $\mathfrak{S}$ is regular if and only if

$$
\Subset\left(\sum_{n=0}^{\infty} a_{n} z^{n}\right)=\sum_{n=0}^{\infty} a_{n} z^{n}
$$

whenever the right-hand side of (3) converges. Analogously, we say that a summability method $\subseteq$ is analytical if for every power series $\sum_{n=0}^{\infty} a_{n} z^{n}$ with nonzero
radius of convergence

$$
\circlearrowleft\left(\sum_{n=0}^{\infty} a_{n} z^{n}\right)=f(z)
$$

whenever the left-hand side of (4) exists $(f(z)$ now being an analytic continuation of $\left.\sum_{n=0}^{\infty} a_{n} z^{n}\right)$. An example of the analytic regular summability method is the wellknown Borel summability method, frequently used in physics (see [M 1] for a recent review). Unfortunately, the use of the Borel summability method is sometimes very limited:

A) The Borel method cannot cope with the horn-shaped singularity, exhibited in the mass QCD ['tH 1], in the "massless" limit of the anharmonic oscillator [Wi], and in some other models. (For recent status of the problem see [KR] and references therein.)

This easily follows from the fact that whenever the Borel sum exists then it defines a function regular in some sector-like neighbourhood of the origin (the convergence of the Borel integral need not be absolute!) [W].

Another shortcoming of the Borel method is the following:

B) In the regular case, i.e. when the series on the right-hand side of (1) has a nonzero radius of convergence, it can in general be analytically continued onto a region of the complex plane which is larger than the region of $z$ for which the Borel sum exists. If the analytic continuation $f(z)$ is not an entire function, the Borel sum may not exist for all $z$ from the Mittag-Leffler (principal) star of $f(z)[\mathrm{H}]$ [further, $\operatorname{MLS}(f)$ ], because always some sector-like domain has to be discarded from the complex plane [SG, M 1].

To illustrate the point $B$ note that the standard Borel method sums the series $\sum_{n=0}^{\infty} z^{n}$ only in the complex halfplane $\operatorname{Re} z<1$ but the analytic continuation $1 /(1-z)$ of the series exists in the whole complex plane except the point $z=1$.

The latter problem has been solved by a new moment constant summability method proposed recently by the author [M1] with the moment sequence $\{\mu(n)\}_{0}^{\infty}$,

$$
\mu(n):=\int_{0}^{\infty} \exp (-\exp t) t^{n} d t
$$



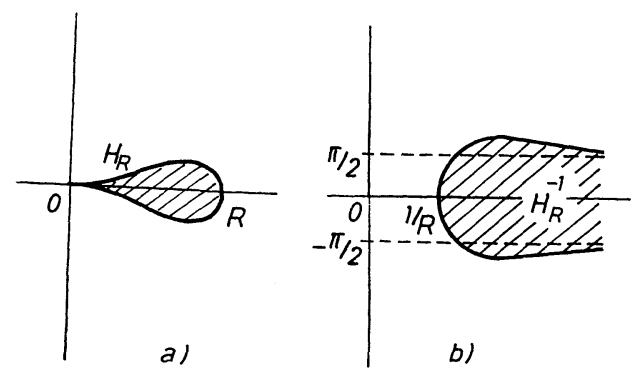

Fig. 1a, b. The horn $H_{R}\left(\right.$ a) and its image $H_{R}^{-1}$ (b) under the mapping $z \rightarrow 1 / z$

Indeed, the main result of [M 1] is that if $f(z)$ is the principal branch of an analytic function regular at the origin, where $f(z)=\sum_{n=0}^{\infty} a_{n} z^{n}$, then the integral $I\left(z_{0}\right)$,

$$
I\left(z_{0}\right)=\int_{0}^{\infty} \exp (-\exp t) \sum_{n=0}^{\infty} a_{n}\left(z_{0} t\right)^{n} / \mu(n) d t,
$$

converges if and only if $z_{0} \in \operatorname{MLS}(f)$, and then

$$
f\left(z_{0}\right)=I\left(z_{0}\right) \text {. }
$$

Thus, the method is analytic and regular. The convergence is absolute and uniform in any bounded subset of $\operatorname{MSL}(f)$ with nonzero distance from the boundary of $\operatorname{MLS}(f)$, and one can also differentiate inside the sign of integration.

In the present paper we will show that this method can be used for the hornshaped singularity as well. We shall deal only with the horn $H_{R}$ (see Fig. 1), defined as

$$
H_{R}:=\{z \mid \operatorname{Re} \omega(1 / z)>\omega(1 / R)\}
$$

where

$$
\omega(z):=\ln F(z), \quad F(z):=\sum_{n=0}^{\infty} z^{n} / \mu(n),
$$

i.e., roughly speaking, with the region of the asymptotic type $(1,1)$ [M 2]. Transition to more general horns of the type $(1, \eta)$ is simply done by mapping $z \rightarrow z / \eta$. The theorems could be modified also for a region of the asymptotic type $(k, \eta)$ with $k>1$. The situation here is, however, more complicated. We shall give also a further improvement of the SAC as discussed in [M2], and show that a product of functions obeying these SAC also obeys the same SAC.

\section{Main Results}

To deal with the singular case let us firstly recall the Nevanlinna theorem [N, So]:

Theorem 1. Let $f(z)$ be analytic in the circle $C_{R}=\{z \mid \operatorname{Re} 1 / z>1 / R\}$, continuous up to the boundary, and satisfy there the estimates

$$
f(z)=\sum_{k=0}^{N-1} a_{k} z^{k}+R_{N}(z)
$$


with

$$
\left|R_{N}(z)\right| \leqq A \sigma^{N} N !|z|^{N}
$$

uniformly in $N$ and in $z \in \bar{C}_{R}$. Then $B(t)$,

$$
B(t):=\sum_{n=0}^{\infty} a_{n} t^{n} / n !,
$$

converges for $|t|<1 / \sigma$ and has an analytic continuation to the striplike region $S_{\sigma}:=\left\{t \mid \operatorname{dist}\left(t, R_{+}\right)<1 / \sigma\right\}$, satisfying the bound

$$
|B(t)| \leqq K \exp (|t| / R)
$$

uniformly in every $S_{\sigma^{\prime}}$ with $\sigma^{\prime}>\sigma$. Furthermore, $f$ can be represented by the absolutely convergent integral

$$
f(z)=(1 / z) \int_{0}^{\infty} \exp (-t / z) B(t) d t
$$

for any $z \in C_{R}$.

Conversely, if $B(t)$ is a function analytic in $S_{\sigma^{\prime \prime}}\left(\sigma^{\prime \prime}<\sigma\right)$ and satisfying there the bound (8), the function $f(z)$ defined by (9) is analytic in $C_{R}$ and satisfies (7) [with $a_{n}=\left.B^{(n)}(t)\right|_{t=0}$ ] uniformly in every $C_{R^{\prime}}^{\prime}$ with $R^{\prime}<R$.

In the next similar role which the exponential function plays in the Nevanlinna theorem will be played by another entire function, $F(t)$, as defined by $(6 \mathrm{~b})$,

$$
F(t)=\sum_{n=0}^{\infty} t^{n} / \mu(n),
$$

and investigated in [M 1]. Indeed, the Nevanlinna-like theorem for the horn $H_{R}$ is as follows.

Theorem 2. Let $f(z)$ be analytic in the horn-shaped region $H_{R}:=\{\operatorname{Re} \omega(1 / z)$ $>\omega(1 / R)\}$, continuous up to the boundary, and satisfy there the estimates

$$
f(z)=\sum_{k=0}^{N-1} a_{k} z^{k}+R_{N}(z)
$$

with

$$
\left|R_{N}(z)\right|<A \mu(N)|z|^{N}
$$

uniformly in $N$ and $z \in \bar{H}_{R}$.

Then

$$
M(t):=\sum_{n=0}^{\infty} a_{n} t^{n} / \mu(n)
$$

converges for $|t|<1$, and has an analytic continuation to the striplike region $S_{1}=\left\{t \mid \operatorname{dist}\left(t, R_{+}\right)<1\right\}$, satisfying the bound

$$
|M(t)| \leqq K \exp [\exp (|t| / R)]
$$

uniformly in every $S_{\varkappa}$ with $x>1$. The analytic continuation of $M(t)$ for $t \in(1, \infty)$ is given as follows,

$$
M(t)=(1 / 2 \pi i) \oint_{\partial H_{R}} F(t / z) f(z) d z / z
$$


Furthermore, $f$ can be represented by the absolutely convergent integral

$$
f(x)=\int_{0}^{\infty} \exp (-\exp t) M(t x) d t
$$

for any $x \in(0, R)$.

The proof of the theorem is rather complicated. Therefore, it is divided into several lemmas. However, the importance of $F(t)$ is already seen from the following argument. If a function $f(z)$ is analytic in the horn $H_{R}$ and continuous in $\bar{H}_{R}$, then for any $x \in H_{R} \cap \mathbb{R}$,

$$
\begin{aligned}
f(x) & =(1 / 2 \pi i) \oint_{\partial H_{R}} f(z) /(z-x) d z \\
& =(1 / 2 \pi i) \oint_{\partial H_{R}} f(z) d z / z \int_{0}^{\infty} \exp (-\exp t) F(t x / z) d t .
\end{aligned}
$$

Note that such a representation of $f(z)$ is impossible by the Borel method. Note also that unlike the disc $C_{R}$ in the Nevanlinna theorem the horn $H_{R}$ is not a starlike region. This is in general the main difference between the regions of the asymptotic type $(0, \eta)$ and $(k, \eta)$ with $k \geqq 1$. This difference causes that by means of moment constant summability methods one cannot recover $f(z)$ from its asymptotic series in the whole horn $H_{R}$ but only for $z \in H_{R} \cap \mathbb{R}$. Physically this is not, however, a problem, since we are expanding in real parameters (couplings).

Before we turn on to the proof of the Nevanlinna-like theorem for the horn $H_{R}$, we shall improve the asymptotic properties of $F(t)$ as given in [M 1] and show that for $|\operatorname{Im} t|>\pi / 2$ and $t$ tending to infinity $F(t)$ uniformly (in $\arg t)$ approaches zero. To prove this we shall need to show that the moment function $\mu(s)$ as defined by (5) exhibits no zero for $\operatorname{Re} s>-1$.

Lemma 1. The function

$$
\mu(s):=\int_{0}^{\infty} \exp (-\exp t) t^{s} d t
$$

1. has a meromorphic extension onto the whole complex plane with simple poles on the negative real axis;

2. its asymptotic behaviour for $s$ tending to infinity, $|\arg s|<\pi$, is governed by the saddle point only and

$$
\mu(s) \sim(2 \pi \ln s / s)^{1 / 2}[\exp -(s / \ln s)][\ln (s / \ln s)]^{s} \quad(s \rightarrow \infty),
$$

where the saddle point is given by the equation

$$
(\exp t) t=s
$$

with the approximate solution

$$
t=\ln (s / \ln s)+\ln \ln s / \ln s+O\left[(\ln \ln s / \ln s)^{2}\right] \quad(s \rightarrow \infty) .
$$

3. The function $\mu(s)$ exhibits no zero for $\operatorname{Re} s>-1$.

Proof. For the proof of 1) and 2) see [M 1]. To prove 3) suppose that for some $s_{0}$,

$$
\mu\left(s_{0}\right)=\int_{-\infty}^{\infty} \exp \left[-\exp (\exp t)+\left(s_{0}+1\right) t\right] d t=0
$$


However, this implies that for any $\delta$,

$$
\int_{-\infty}^{\infty} \exp \left\{-\exp [\exp (t+\delta)]+\left(s_{0}+1\right) t\right\} d t=0,
$$

which means, on the other hand, that

$$
\int_{-\infty}^{\infty} \exp \left[-\exp (\exp t)+\left(s_{0}+2\right) t\right][-\exp (\exp t)] d t=0 .
$$

Repeating this procedure $m$-times one gets that

$$
\begin{aligned}
I(m):= & \int_{0}^{\infty} \exp \left[-\exp t+m t+\left(s_{0}+m\right) \ln t\right] \\
& \times A^{-m}[A(-1+d / d A)]^{m-1}(-A) d t=0,
\end{aligned}
$$

where $A=\exp t$. Note that the term $D(t)$,

$$
D(t):=A^{-m}[A(-1+d / d A)]^{m-1}(-A),
$$

is bounded on $t \in(0, \infty)$ and nonzero for sufficiently large $t$. On the other side, if $m$ is sufficiently large we may use an asymptotic formula to evaluate $I(m)$. Thus,

$$
I(m) \sim\left[2 \pi u^{\prime} u /(u+1)\right]^{1 / 2} \exp \left[-\exp u+m u+\left(s_{0}+m\right) \ln u\right] D(u) \neq 0,
$$

since $u(m)$ is the solution of the equation

$$
-e^{t}+m+\left(s_{0}+m\right) / t=0,
$$

and which behaves like

$$
u(m) \sim \ln \left[m+\left(s_{0}+m\right) / \ln m\right] \quad(m \rightarrow \infty) . \quad \text { Q.E.D. }
$$

Throughout the paper we shall frequently use also the following expression of the asymptotic behaviour of $\mu(s)$ for $s$ tending to infinity and $|\arg s|<\pi$,

$$
\mu(s) \sim\left[2 \pi w(s) w^{\prime}(s)\right]^{1 / 2} \exp [-\exp w(s)+n \ln w(s)],
$$

where $w(s)$ denotes the exact solution to the equation $(17 \mathrm{a})$, and $w^{\prime}(s) \sim 1 / s-1 / s w(s)$ when $s$ tends to infinity and $|\arg s|<\pi$.

Lemma 2. Let $\{\mu(n)\}_{0}^{\infty}$ be the Stieltjes moment sequence generated by the measure $\exp (-\exp t) d t(5)$. Then the function $F(t)$,

$$
F(t)=\sum_{n=0}^{\infty} t^{n} / \mu(n)
$$

is an entire function with the following asymptotic behaviour at infinity:

1) For $|\operatorname{Im} t| \leqq \pi / 2$ the asymptotic behaviour is determined by the saddle point

$$
s=(\exp t) t
$$

and

$$
F(t) \sim(t+1) \exp (t+\exp t) \quad(t \rightarrow \infty)
$$

2) For $|\operatorname{Im} t|>\pi / 2$,

$$
|F(t)| \leqq O\left(|t|^{\sigma}\right) \quad(t \rightarrow \infty) .
$$

$\sigma$ being some constant, $-1<\sigma<0$. 
Proof. The statement of the lemma is proved in [M1] for $\sigma$ sufficiently large. By virtue of the improved Lemma 1 we can readily repeat the proof of the lemma with modified integral representation of $F(t)$ (Euler-Maclaurin sum formula) [F, J],

$$
F(t)=\int_{\sigma}^{\infty} \exp (s \ln t) / \mu(s) d s+O\left(|t|^{\sigma}\right) \quad(t \rightarrow \infty),
$$

where $\sigma$ is now from the interval $(-1,0)$. Q.E.D.

We are in the position to turn to the proof of Theorem 2 now. We shall follow Sokal's strategy of the proof of the Nevanlinna theorem [So]. Lemma 2 provides us with an integral representation of the monomials $t^{n} / \mu(n)$ for any positive $n$ and $t \geqq 1$,

$$
(1 / 2 \pi i) \oint_{\partial H_{R}} F(t / z) z^{n} d z / z=t^{n} / \mu(n),
$$

where the integral is taken counterclockwise along the boundary of $H_{R}$. The formula will be used to express $M(t)$ [an analogue of $B(t)$ ] in terms of $f(z)$. To find the minimal domain of analyticity of $M(t)$ we shall need a bound on $F^{(m)}\left(z_{0}\right)$ for $m$ tending to infinity (Lemma 3). Finally, after the Lemma 4 we shall give all the lemmas together and complete the proof of the theorem.

Lemma 3. For any $z_{0}$ such that $\operatorname{Re} z_{0}>0$ and $n>0$,

$$
\begin{aligned}
\left|F^{(n)}\left(z_{0}\right)\right|< & \text { const } \exp \left[x_{0}\left(n / \ln n+n / \ln ^{2} n\right)+2 \ln w(n)+O(1 / w(n))\right] \\
& \times\left|F\left(z_{0}\right)\right| n ! / \mu(n),
\end{aligned}
$$

where $x_{0}=\operatorname{Re} z_{0}$.

Proof. Firstly,

$$
\begin{aligned}
F^{(n)}\left(z_{0}\right) / n ! & =(1 / 2 \pi i) \oint_{C} F\left(z_{0}+z\right) / z^{n+1} d z \\
& =(1 / 2 \pi i) \oint_{C} \exp \left[\omega\left(z+z_{0}\right)-n \ln z\right] d z / z,
\end{aligned}
$$

where $C$ is a simple contour enclosing the origin. The saddle point of the integral is a solution to the equation (using Lemma 2)

$$
z \exp \left(z_{0}+z\right)+z+z /\left(z_{0}+z+1\right)=n \text {. }
$$

Denote the solution $v(n)$. By virtue of the Appendix we have,

$$
v(n)=w(n)-z_{0}-z_{0} /[w(n)+1]+w(n) w^{\prime}(n)+O\left[w^{\prime}(n)\right] \quad(n \rightarrow \infty) .
$$

Note that $z_{0}+v(n)$ approaches the real positive axis when $n \rightarrow \infty$, and a part of the contour of the steepest descent nearby the saddle point is approximately a segment of the circle centered at $z_{0}$. After the saddle point evaluation of the integral one finds that

$$
F^{(n)}\left(z_{0}\right) / n ! \sim\left[v(n) v^{\prime}(n) / 2 \pi\right]^{1 / 2} \exp \left[\omega\left(z_{0}+v(n)\right)-n \ln v(n)\right]
$$

or

$$
\begin{aligned}
& F^{(n)}\left(z_{0}\right) \mu(n) / n ! \sim \text { const } \exp \left\{z_{0}[n /(w(n)+1)+n /[w(n)(w(n)+1)]\right. \\
&+2 \ln w(n)+O(1 / w(n))\} \quad(n \rightarrow \infty),
\end{aligned}
$$


respectively. On the other hand, if we consider the integral on the right-hand side of (20) for $n$ fixed and $z_{0}$ tending to infinity then we have,

$$
\left|F^{(n)}\left(z_{0}\right) / n !\right| \leqq \exp \operatorname{Re}\left(z_{0}+1\right) .
$$

Giving (18), (22), and (23) together and using the approximate expression (17b) for $w(n)$ one finally gets the statement of the lemma. Q.E.D.

Lemma 4. Let $J_{x}(z)$ be the integral

$$
\begin{aligned}
J_{x}(z): & =\int_{0}^{1} \exp [-\exp (t / x)] F(t z) d t \\
& =\sum_{n=0}^{\infty}(x z)^{n} / \mu(n) \int_{0}^{1 / x} \exp (-\exp t) t^{n} d t
\end{aligned}
$$

Then we have

$$
(1 / 2 \pi i) \oint_{\partial H_{R}} J_{x}(1 / z) z^{n} d z / z=x^{n} / \mu(n) \int_{0}^{1 / x} \exp (-\exp t) t^{n} d t,
$$

where the integral is taken counterclockwise along the boundary of $H_{R}$.

Proof. Note that $x^{n} \int_{0}^{1 / x} \exp (-\exp t) t^{n} d t$ behaves like $\exp [-\exp (1 / x)] /(n x)$ when $n$ tends to infinity so that one expects that $x$ will only produce an overall factor to the asymptotic behaviour of $J_{x}(z)$ when $z$ tends to infinity. This can be confirmed as in Lemma 2. One uses the Euler-Maclaurin sum formula and finds that for $|\operatorname{Im} z|$ $\leqq \pi / 2$ the asymptotic behaviour is governed by a saddle point, which is here determined by the equation

$$
\ln w(s)+1 / s=\ln z
$$

Thus, the contribution $V_{s}$ of the saddle point is

$$
V_{s}=(z+1) \exp \{\exp z[\exp (-\exp (-z) / z)]+1\} / x \quad(z \rightarrow \infty) .
$$

By analogy with Lemma 2 one can easily prove that for $z$ tending to infinity and $|\operatorname{Im} z|>\pi / 2$ function $J_{x}(z)$ tends to zero in the same manner as $F(z)$. The proof of the statement of the lemma is then trivial. Note that the integral from $J_{x}(z) / z$ along a segment of $\partial H_{R}^{-1}$ which starts at infinity and terminates at some $z_{0}$ on the contour converges even absolutely.

Proof of the Theorem.

i) Under the hypotheses of the theorem one easily proves that the series (11) converges for $|t|<1$. Let us consider the integral $d(t)$.

$$
d(t):=(1 / 2 \pi i) \underset{\partial H_{R}}{\oint} F(t / z) f(z) d z / z,
$$

where $t \geqq 1$. Two remarks are in order. In contrast to the Nevanlinna theorem one cannot use the integral on the right-hand side of (24) for $0<t<1$, as it is not possible to satisfy both conditions that the contour of integration in (19) be the contour which tends to zero on the boundary of a $t H_{r}$ for some $r$, and at the same 
time lie in $H_{R}$. Whenever $t \notin \mathbb{R}$ the integral is identically zero (by virtue of Lemma 2). Hence, it cannot yield an analytic continuation of $d(t)$ for $t \notin \mathbb{R}$.

From the properties of $F(z)$ it is immediately seen that the integral on the righthand side of (24) converges for $t>1$ absolutely and uniformly on any closed subset of $(1, \infty)$. For $t=1$ the integral converges by virtue of the Abel-Dirichlet lemma. Hence, $d(t)$ is a $C^{\infty}$ function on the interval $(1, \infty)$ and possesses the right derivatives at the point $t=1$.

To prove that the series (11) converges at $t=1$, we make use of Lemma 3 , and we rewrite $d(1)$ as follows,

$$
d(1)=\sum_{k=0}^{N-1} a_{k} / \mu(k)+(1 / 2 \pi i) \oint_{\partial H_{R}} F(1 / z) R_{N}(z) d z / z .
$$

As the integration on the right-hand side of (25) runs along the boundary of $H_{R}$ on which $\operatorname{Re} \omega(1 / z)=$ const $(=\omega(1 / R))$ the integral can be bounded from above as follows:

$$
\begin{aligned}
& \left|(1 / 2 \pi i) \oint_{\partial H_{R}} F(1 / z) R_{N}(z) d z / z\right| \\
& \leqq(A / \pi) \exp (\omega(1 / R)) \mu(N) \int_{\partial H_{R}^{+}} \exp (N \ln r) d r / r \\
& =(\mathrm{A} / \pi) \exp (\omega(1 / R)) \mu(N) \exp (-N \ln (1 / R)) / N \text {, }
\end{aligned}
$$

where $\partial H_{R}^{+}$means that we integrate along $\partial H_{R}$ in the first quadrant. After distorting the contour of integration up $\partial H_{r}$ with $r=1 / w(N)$ we have,

$$
\left|(1 / 2 \pi i) \oint_{\partial H_{R}} F(1 / z) R_{N}(z) d z / z\right| \leqq\{A \sqrt{2 w(N) / \pi N} \rightarrow 0 \quad(N \rightarrow \infty)
$$

Thus, $M(1)=d(1)$. The same is true also for derivatives, i.e. $M^{(n)}\left(1_{-}\right)=d^{(n)}\left(1_{+}\right)$for any $n \in \mathscr{N}$. Indeed, if $f(z)$ satisfies SAC then the same SAC will satisfy also its derivatives $f^{(n)}(z)$ (may be in a horn $H_{R}$ with different $R$ ). So

$$
d^{(n)}\left(1_{+}\right)=\sum_{k=0}^{N-1} c_{k} / \mu(k)+(1 / 2 \pi i) \oint_{\partial H_{R}} F(1 / z) R_{N}^{(n)}(z) d z / z,
$$

where $c_{k}=(k+n)(k+n-1) \ldots(k+1) a_{k+n}$, and the integral can be estimated in the same manner as above.

To determine the minimal region of analyticity we express $d^{(n)}(t)$ as follows:

$$
d^{(n)}(t)=n ! a_{n} / \mu(n)+1 /(2 \pi i) \oint_{\partial H_{R}} F^{(n)}(t / z) R_{n+1}(z) z^{-n} d z / z
$$

Now, using Lemma 3 one easily finds that

$$
\begin{aligned}
\left|d^{(n)}(t)\right| & \leqq \text { const } F(t / R) n ! \\
& \times \exp \left[(t / R)\left(n / \ln n+n / \ln ^{2} n\right)+2 \ln w(n)+O(1 / w(n))\right] .
\end{aligned}
$$

One may justify, that

$$
\sum_{n=0}^{\infty} d^{(n)}\left(t_{1}\right)\left(t-t_{1}\right)^{n} / n !=\sum_{n=0}^{\infty} d^{(n)}\left(t_{2}\right)\left(t_{2}-t\right)^{n} / n !,
$$

whenever $1<t_{1} \leqq t \leqq t_{2}$, and $\left|t-t_{i}\right|<1$, where $i=1,2$. 


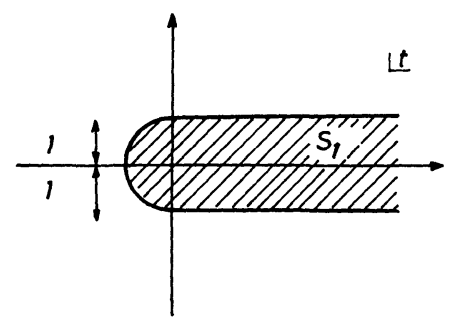

Fig. 2. Minimal region of analyticity of $M(t)$

Thus $M(t)$,

$$
M(t)=\left\{\begin{array}{lr}
\sum_{n=0}^{\infty} a_{n} t^{n} / \mu(n), & -1<t \leqq 1 ; \\
d(t), & t \geqq 1 ;
\end{array}\right.
$$

is shown to be analytic at least in the striplike region $S_{1}$ (see Fig. 2).

Bound (12) on $M(t)$ follows immediately from the relation (26).

ii) To prove the relation (14), let us consider the integral

$$
1 / x \int_{0}^{\infty} \exp [-\exp (t / x)] M(t) d t
$$

for any $x \in(0, R)$. The integral,

$$
\int_{1}^{\infty} \exp [-\exp (t / x)](1 / 2 \pi i) \oint_{\partial H_{R}} f(z) F(t / z) d z / z d t,
$$

is absolutely convergent. Therefore, the right-hand side of (14) [the integral (27)] can be recasted into the form,

$$
\begin{aligned}
& f(x)-(1 / 2 \pi i x) \underset{\partial H_{R}}{ } J_{x}(1 / z) f(z) d z / z \\
& \quad+1 / x \sum_{n=0}^{\infty} a_{n} / \mu(n) \int_{0}^{1} \exp [-\exp (t / x)] t^{n} d t .
\end{aligned}
$$

Using the Lemma 4 one finds that the last two terms cancel each other. Indeed,

$$
\begin{aligned}
1 /(2 \pi i) \oint_{\partial H_{R}} J_{x}(1 / z) f(z) d z / z \\
=\sum_{n=0}^{N-1} a_{n} / \mu(n) \int_{0}^{1} \exp [-\exp (t / x)] t^{n} d t \\
\quad+1 /(2 \pi i) \oint_{\partial H_{R}} J_{x}(1 / z) R_{N}(z) d z / z .
\end{aligned}
$$

To optimize the bound on the integral on the right-hand side we choose the contour of integration to be $\partial H_{1 / w(N)}$. Then, in virtue of $(17 \mathrm{a}, \mathrm{b}, 18)$,

$$
\left|1 /(2 \pi i) \oint_{\partial H_{R}} J_{x}(1 / z) R_{N}(z) d z / z\right| \leqq \frac{A}{x} \sqrt{2 w(N) / \pi N} \rightarrow 0 \quad(N \rightarrow \infty) .
$$




\section{Corollary. Let}

1. $M(t):=\sum_{n=0}^{\infty} a_{n} t^{n} / \mu(n)$ has nonzero radius of convergence and can be analytically extended on the real positive axis;

2. $|M(t)|<$ const $\exp (\exp (t / R))$ on the axis.

Then

$$
f(x):=1 / x \int_{0}^{\infty} \exp (-\exp (t / x)) M(t) d t
$$

converges absolutely for $x \in(0, R)$ and

$$
f(x) \sim \sum_{n=0}^{\infty} a_{n} x^{n} \quad\left(x \rightarrow 0_{+}\right) .
$$

Proof. Use the Laplace method to evaluate the integral.

Now, after the proof of the Theorem 2 we want to show an important property of the class $\mathfrak{h}$ of function which obey the hypotheses of the theorem.

Lemma 5. The class of function $\mathfrak{h}$ is closed under product, i.e. if $f_{1}(z)$ and $f_{2}(z)$ are two functions from $\mathfrak{h}$, then $g(z):=f_{1}(z) \cdot f_{2}(z) \in \mathfrak{h}$.

Proof. To prove the lemma it is sufficient to prove that

$$
\lim _{N \rightarrow \infty} \sum_{k=0}^{N} \mu(N-k) \mu(k) / \mu(N)<\infty \text {. }
$$

From the Lemma 1 one can derive that if $N$ is sufficiently large then for $q<N / 2$,

$$
\mu(N-q) / \mu(N) \sim \exp \left[-q \ln \ln N-q / N-\left(q+q^{2}\right) /(N \ln N)\right] .
$$

Thus, if $j$ is such that the asymptotic formula to estimate $\mu(s)$ for $s \geqq j$ can be used, then

$$
\begin{aligned}
& \sum_{k=0}^{N} \mu(N-k) \mu(k) / \mu(N) \leqq 2 \sum_{k=0}^{\left[\frac{N}{2}+1\right]} \mu(N-k) \mu(k) / \mu(N) \\
& \quad \text { const } \sum_{k \geqq j}^{\left[\frac{N}{2}+1\right]} \exp \{-\ln \ln N[\ln k / \ln \ln N+k[1-\ln \ln k / \ln \ln N]\},
\end{aligned}
$$

where the series on the right-hand side converges in the limit $N \rightarrow \infty$, since its last term behaves like $1 /[N \exp (N \ln 2 / \ln N)]$. Q.E.D.

\section{Conclusion}

We have just shown that the summability method recently proposed in [M 1], where it was studied in the regular case, can deal with the horn-shaped singularity as well. To our knowledge it is the first one with this property. In $[M 1,2]$ the advantages and shortcomings of the method have been discussed, and also applications to the Rayleigh-Schrödinger perturbation theory [K, RS 78] and the derivative analyticity relations $[\mathrm{FK}]$ have been shown. 
Like any analytic regular summability method also our method may have a wide domain of applications. We will not give a list of them because the reader can easily judge whether it is interesting for him or not.

If additional information is available, the method, like the Borel one, can be made more powerful when combined with conformal mapping. It is also obvious that the use of the method is not confined to perturbation expansion in coupling but it works as well for any kind of power expansion, like the $1 / N$ expansion etc.

We note that Theorem 2 gives a generalization of SAC as proved in [M2]. Indeed, by the theorem a function cannot exist which

i) is analytic in the horn $H_{R}$ and continuous up to the boundary;

ii) possesses there the asymptotic expansion (10) which has equal sign coefficients $a_{n}$ for $n \geqq n_{0}$.

We would like to point out that assertions of the type that a quantity $Q_{1}$ equals another quantity $Q_{2}$ to all orders of perturbation theory are very vague till the $\mathrm{SAC}$ are shown to be valid. The SAC can also be used as a criterion to look for the true ground state of a theory [M 2].

Finally, our method gives a generalization of [S] since Theorem 2 together with Lemma 5 provide a summability mechanism which apart from invariance conditions and linear covariances preserves also nonlinear perturbative conditions such as unitarity of the Feynman series.

An open question still remains how the summability properties (both Borel's and ours) are transported in equations such as the Dyson-Schwinger equations for Green's functions ['tH 2]. Another important question is whether one could encounter a situation that the perturbative series has slower than the factorial growth and its coefficients obey the bound (10). The answer to the last question seems to be affirmative at least for the theory for which the maximal region of analyticity in an expansion parameter is a horn, as an adequate perturbation scheme should obey SAC and give unambiguous results. It is worth remembering that the factorial growth of perturbation theory was proved especially for bosonic theories while inclusion of fermions seems to slow the divergence down (see e.g. $[\mathrm{P}]$ ). Moreover, sometimes the factorial growth is an artefact of approximation used and a more careful analysis may give even a convergent result [C]. A convergent perturbation expansion can also be obtained when instead of the expansion in renormalized coupling constant one uses the expansion in powers of the running coupling constant (see e.g. [Po, T] and references therein). In many cases the method can be successfully applied to the regularized theory by setting it on the lattice, etc. $[\mathrm{Fe}, \mathrm{S} 1, \mathrm{Se}]$.

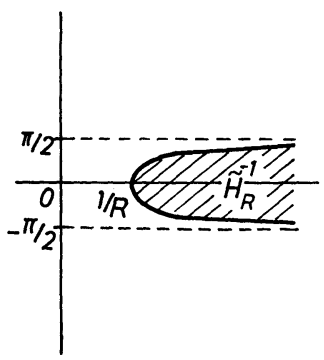

Fig. 3. The image $\widetilde{H}_{R}^{-1}$ of the horn $\tilde{H}_{R}$ under the mapping $z \rightarrow 1 / z$ 
One could get also analogous results by other slightly modified Stieltjes moments like

$$
\tilde{\mu}(n):=\int_{0}^{\infty} \exp (t-\exp t) t^{n} d t,
$$

etc. In this case one only needs to replace $\omega(z)$ by $\tilde{\omega}(z)=\exp z+\ln (z+1)$ and $H_{R}$ by $\widetilde{H}_{R}:=\{\operatorname{Re} \tilde{\omega}(1 / z)>\tilde{\omega}(1 / R)\}$. Note that in contrast to the previous case the boundary $\partial \widetilde{H}_{R}^{-1}$ of $\widetilde{H}_{R}^{-1}$ approaches the straight line $\operatorname{Im} z=\pi / 2$ from below (see Fig. 3).

\section{Appendix}

In this appendix we express the solution $v(n)$ to Eq. (21),

$$
v(n) \exp \left(z_{0}+v(n)\right)+v(n)+v(n) /\left(v(n)+z_{0}+1\right)=n,
$$

in terms of $w=w(n)$, the solution to Eq. (17a),

$$
w(n) \exp w(n)=n .
$$

We take first logarithms of these equations. Upon the substitution $v(n)$ $=w(n)-z_{0}-\delta$ and some manipulations we have,

$$
-\delta+\ln \left[\delta+z_{0} /(w+1)\right]=\ln w w^{\prime}+1 / w+\left(z_{0}+\delta\right) / w^{2}+O\left(w^{3}\right) .
$$

Thus,

$$
\delta+z_{0} /(w+1)=w w^{\prime}+\left[1 / w-z_{0} /(w+1)\right] w w^{\prime}+O\left[\left(w w^{\prime}\right)^{2}\right] \quad(n \rightarrow \infty),
$$

i.e. $\delta=O(1 / \ln n)$ when $n$ tends to infinity.

Acknowledgements. I am indebted J. Fischer for continuous interest in my work and valuable comments and especially to V. Šverák for many stimulating discussions. I thank also J. Magnen, G. 't Hooft, and A. S. Wightman for encouraging correspondence.

\section{References}

[C] Caianiello, E.R.: Number of Feynman graphs and convergence. Il Nuovo Cim. 3, 223-225 (1956)

[F] Fedoriuk, M.V.: Asymptotic estimates: Integrals and series (in Russian). Moscow: Nauka 1987

[Fe] Feldman, J., Magnen, J., Rivasseau, V., Sénéor, R.: Massive Gross-Neveu model: a rigorous perturbative construction. Phys. Rev. Lett. 54, 1479-1481 (1985)

[FK] Fischer, J., Kolář, P.: Differential forms of the dispersion integral. Czech. J. Phys. B 37, 297-310 (1987)

[H] Hardy, G.: Divergent series. Oxford: Oxford University Press 1949

[J] Jevgrafov, M.A.: Asymptotic estimates and entire functions (in Russian). Moscow: Nauka 1979

[K] Kato, T.: Perturbation theory for linear operators, 2nd ed. Berlin, Heidelberg, New York: Springer 1976

[KR] Khuri, N.N., Ren, H.C.: Explicit solutions for the running coupling constant and the separatrix of quantum field theory. Ann. Phys. (NY) 189, 142-154 (1989) 
[M 1] Moroz, A.: Novel summability method generalizing the Borel method. Czech. J. Phys. B 40, 705-726 (1990)

[M2] Moroz, A.: Strong asymptotic conditions for horn-shaped regions (submitted for publication)

[N] Nevanlinna, F.: Zur Theorie der asymptotischen Potenzreihen. PhD thesis of the Alexander University, Helsingfors 1918

[P] Parisi, G.: Asymptotic estimates in perturbation theory with fermions. Phys. Lett. 66 B, 382-384 (1977)

[Po] Pordt, A.: Renormalization theory for use of convergent expansions of euclidean quantum field theory. In:'t Hooft, G. et al. (eds.) Nonperturbative quantum field theory. Proceedings, Cargése 1987, pp. 503-511. New York, London: Plenum Press 1988

[R] Ritt, J.F.: On the derivatives of a function at a point. Ann. Math. 15, 18-23 (1916)

[RS] Reed, M., Simon, B.: Methods of modern mathematical physics, Vol. IV. Analysis of operators. New York, San Francisco, London: Academic Press 1978

[SG] Sansonne, G., Gerretsen, J.: Lectures on the theory of functions of a complex variable, Vol. I. Noordhoff, Groningen, The Netherlands 1960

[S] Simon, B.: Summability methods, the strong asymptotic condition, and unitarity in quantum field theory. Phys. Rev. Lett. 28, 1145-1146 (1972)

[S1] Simon, B.: Convergence of regularized, renormalized perturbation series for superrenormalizable field theories. Il Nuovo Cim. 59, 199-214 (1969)

[Se] Seiberg, N.: Topology in strong coupling. Phys. Rev. Lett. 53, 637-640 (1984)

[So] Sokal, A.D.: An improvement of Watson's theorem on Borel summability. J. Math. Phys. 21, 261-263 (1980)

[T] Timme, H.-J.: Convergent perturbation expansions in irrelevant interactions for the renormalization group flow of a four-dimensional hierarchical $S U(2)$ lattice gauge field model. Hamburg preprint DESY 89-110

['t H 1] 't Hooft, G.: Can we make sense out of "quantum chromodynamic"? In:Zichchi, A. (ed.) The whys of subnuclear physics. Proceedings, Erice 1977, pp. 943-971. New York, London: Plenum Press 1979

['t H 2] t' Hooft, G.: Private correspondence

[W] Widder, D.V.: The Laplace transform. Princeton, NJ: Princeton University Press 1946

[Wi] Wightman, A.S.: Should we believe in quantum field theory? In: Zichichi, A. (ed.) The Whys of subnuclear physics. Proceedings, Erice 1977, pp. 983-1015. New York, London: Plenum Press 1979

Communicated by K. Gawedzki 